\title{
Sintonización genética de sistema de inferencia difuso aplicado al control de posición en un motor de corriente directa
}

\section{Genetic tuning of fuzzy inference system applied to position control in a direct current motor}

RODRÍGUEZ-FRANCO, Martín Eduardo †*־,”, DELGADO-GUERRERO, Sergio Humberto', LÓPEZ-ÁLVAREZ, Yadira Fabiola’ y JARA-RUIZ, Ricardo'

'Universidad Tecnológica del Norte de Aguascalientes, México.
"Universidad Politécnica de Aguascalientes, México.

ID $1^{\text {er }}$ Autor: Martín Eduardo, Rodríguez-Franco / ORC ID: 0000-0002-6804-4777, Researcher ID Thomson: T-15392018, CVU CONACYT ID: 660892

ID $1^{\text {er }}$ Coautor: Sergio Humberto, Delgado-Guerrero / ORC ID: 0000-0003-2521-5887, Researcher ID Thomson: V-17472018, CVU CONACYT ID: 240475

ID $2^{\text {do }}$ Coautor: Yadira Fabiola, López-Álvarez / ORC ID: 0000-0002-9041-1908, Researcher ID Thomson: T-1555-2018, CVU CONACYT ID: 375952

ID $3^{\text {er }}$ Coautor: Ricardo, Jara-Ruiz / ORC ID: 0000-0001-7725-4138, Researcher ID Thomson: T-1532-2018, CVU CONACYT ID: 630276

DOI: $10.35429 /$ JSI.2020.14.4.1.6

Recibido Abril 10, 2020; Aceptado Junio 29, 2020

\section{Resumen}

El presente trabajo propone el desarrollo y la aplicación de un algoritmo genético para mejorar la función de una máquina de inferencia difusa empleada en el control de posición en el eje de un motor de corriente directa. En esta primera fase, el estudio se realiza sobre el sistema dejando de lado la función de transferencia de que define las características dinámicas del actuador mencionado, lo que lleve a la posterior comprobación física de su comportamiento. Se plantea el análisis del error calculado como la diferencia entre la posición deseada y la posición real alcanzada por eje del motor, así como de la señal que el sistema de inferencia habrá de generar para corregir la magnitud de error dada. Asimismo, se exponen los fundamentos teóricos que sustentan a esta aplicación y la metodología ejecutada para la obtención de los resultados que constatan la efectividad en el empleo del algoritmo genético como medio de sintonización del sistema de inferencia difuso desarrollado, al describir un error global inferior al $20 \%$ en la acción de control deducida con respecto a la deseada.

Lógica difusa, Algoritmo genético, Sintonización de control de posición

\begin{abstract}
This paper proposes the development and application of a genetic algorithm to improve the function of a fuzzy inference machine used in the shaft position control of a direct current motor. In this first stage, the study is executed on system leaving out the transfer function that defines the dynamic characteristics of the mentioned actuator, which leads to the subsequent physical verification of its behavior. The analysis of the calculated error is considered as the difference between the desired position and the current position reached by motor shaft, as well as the signal that the inference system will generate to correct the given magnitude of error. Likewise, the theoretical foundations that support this application and the methodology executed to obtain the results that confirm the effectiveness in the use of the genetic algorithm as a means of tuning the fuzzy inference system developed are described, when describing a global error lower than $20 \%$ in the control action deducted with respect to the one desired.
\end{abstract}

Fuzzy logic, Genetic algorithm, Position control tuning

Citación: RODRÍGUEZ-FRANCO, Martín Eduardo, DELGADO-GUERRERO, Sergio Humberto, LÓPEZ-ÁLVAREZ, Yadira Fabiola y JARA-RUIZ, Ricardo. Sintonización genética de sistema de inferencia difuso aplicado al control de posición en un motor de corriente directa. Revista de Innovación Sistemática. 2020. 4-14:1-6.

*Correspondencia al Autor (Correo Electrónico: martin.rodriguez@utna.edu.mx)

$\dagger$ Investigador contribuyendo como primer autor. 


\section{Introducción}

La lógica difusa es empleada para describir fenómenos del mundo real cuyas características principales son la vaguedad y la ambigüedad (Czabanski, Jezewski \& Leski, 2017). Es a partir de uso de un lenguaje natural que son introducidas reglas a un controlador, refiriéndose a sistemas de control, o computadora para que éstos imiten la forma del razonamiento humano, en el cual, la esencia del análisis sobre una característica de interés es lingüística y no matemática (Zadeh, 1999). Así, un sistema de inferencia difuso emplea tales variables lingüísticas para conformar una base de conocimiento que permite discernir un comportamiento de salida, dados ciertos parámetros de entrada; ambos con una pertenencia no absoluta a estos conjuntos (Kesarwani \& Khilar, 2019).

Sin embargo, para la descripción de un fenómeno real la aplicación de variables lingüísticas queda limitada, por lo que resulta necesaria la adecuada interpretación tanto a la entrada como a la salida del sistema de análisis (Bede, Rudas \& Fodor, 2007). Con lo cual, se dota al controlador de elementos que le permitan traducir la información adquirida de la naturaleza para su procesamiento, y posterior a éste, sea capaz de devolver de forma precisa la acción que se tomará para corrección o regulación de un comportamiento dado (Qiao, et al., 2004) (Lee, et al., 2010). Es así, que el diseño de controladores mediante lógica difusa habrá de tomar como referencia el conocimiento previo que se posea del elemento al cual será aplicado (Kunqi, et al., 2019). Con el fin de obtener la respuesta adecuada en un sistema de inferencia difuso, se contempla el uso de algún algoritmo que permita la búsqueda y correlación de características del elemento bajo estudio (Passos \& Barrenechea, 2019).

En la presente aplicación, se emplean algoritmos genéticos para el entrenamiento del sistema de inferencia desarrollado, lo que permite la optimización del proceso de búsqueda de parámetros para su ajuste (Arthur, et al., 2019). Los algoritmos genéticos se inspiran en la evolución biológica de las especies a partir del proceso de selección natural, fenómeno estudiado por el naturalista inglés Charles Darwin (Passos \& Barrenechea, 2019).
La hibridación entre lógica difusa y algoritmos genéticos permite la convergencia eficiente de la respuesta del sistema en una solución eficaz dentro de un espacio de búsqueda acotado, estableciendo no sólo una solución local óptima, sino global (Civelek, 2019).

\section{Metodología}

La presente aplicación propone la conformación de un prototipo de sistema de control en lazo cerrado de posición, cuyo actuador es un motor de corriente directa, mismo que estará bajo la acción de un controlador difuso. El único parámetro contemplado para este proceso es la señal del error deducida entre un valor de consiga dado por el usuario y aquel leído a partir de la posición real alcanzada por el prototipo. Mientras la variable emitida por el controlador será la cantidad de energía gestionada hacia el motor empleado, con la finalidad de promover un cambio en la posición real de su eje. Se trata de una señal eléctrica del tipo PWM (pulse width modulation) correspondiente a la magnitud del cambio sugerido por el controlador, una vez procesado el error.

Para asegurar una adecuada funcionalidad del controlador empleado se combinan las técnicas de lógica difusa y algoritmos genéticos. Es a partir de la propuesta de conjuntos difusos asociados al parámetro y la variable de proceso, que se realiza una relación de implicación entre éstos, para definir la acción y salida de control más eficiente, dadas las características del error deducido. A su vez, con la finalidad de establecer una respuesta de control en el sistema de posición, similar a la otorgada a partir del controlador difuso, se emplean algoritmos genéticos para su sintonización. Un conjunto de valores de entrada y salida procedentes del sistema difuso serán los parámetros para el entrenamiento del algoritmo genético utilizado, el cual buscará aproximar las magnitudes y la forma de los conjuntos de entrada y salida del controlador difuso; sin que exista otro medio de relación entre la funcionalidad de ambos. 
Posteriormente, se realizará una comparación entre los comportamientos del sistema de control a partir de la función del controlador difuso originalmente propuesto y aquel cuyos elementos fueron deducidos mediante el uso de algoritmos genéticos, empleando datos distintos a los utilizados para el entrenamiento de éste. Se pretende caracterizar un comportamiento muy aproximado entre las respuestas obtenidas por la aplicación de ambas técnicas, lo que lleve a constatar el uso de algoritmos genéticos para la deducción de parámetros y variables de operación de un sistema difuso, con una fidelidad competitiva.

Cabe mencionar que para fines del presente análisis la respuesta del sistema de control establecida a partir de su dinámica, y representada a través de la función de transferencia respectiva, no es contemplada. Es, por tanto, que la orientación de este estudio será la validación del uso de algoritmos genéticos como un medio factible para la adecuada sintonización de controladores difusos, a partir de la información aportada por datos de entrada y salida recopilados de la operación física del proceso bajo análisis. Un análisis distinto al expuesto promoverá brindar continuidad al presente trabajo.

\section{Proceso de experimentación}

Este análisis se aplica a un sistema de control de posición de un motor de corriente directa, la consigna se ingresa al sistema a partir de la señal dada por un primer potenciómetro manipulado por el usuario; mientras un segundo potenciómetro, unido al eje del motor, retroalimenta la posición obtenida, como se expone en la figura 1. Ambas señales son ingresadas a una tarjeta controladora Arduino UNO a través de puertos analógicos de entrada con resolución a 10 bits. A partir del tratamiento de los datos ingresados, se emiten dos señales de control hacia una etapa de potencia (atendiendo al sentido de giro requerido), y de ahí al motor, empleando puertos de salida analógica con resolución a 8 bits.

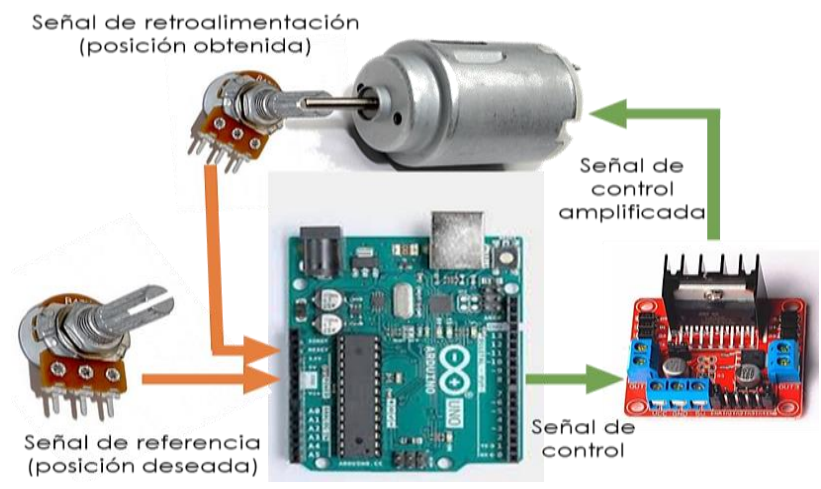

Figura 1 Sistema de control de posición de motor de corriente directa y señales

Fuente: elaboración propia

La magnitud del error, es decir, la diferencia entre los valores de posición deseada y obtenida, se determina dentro del controlador. Se contempló un sistema de inferencia difuso con cinco conjuntos de entrada y cinco conjuntos de salida. El primer grupo corresponde al error (negativo relevante, negativo poco relevante, nulo, positivo poco relevante $\mathrm{y}$ positivo relevante), y el segundo a la acción de control (negativa relevante, negativa poco relevante, nula, positiva poco relevante $\mathrm{y}$ positiva relevante), generada dado el análisis del error. Los límites de la señal de error se establecen en (1), mientras los correspondientes a la señal de control emitida se definen en (2). Ambos conjuntos se muestran en los gráficos 1 y 2 , respectivamente.

error $=\{$ input $\in R \mid$ input $=[-100,100]\}$

control $=\left\{\right.$ output $\in R \mid$ output $\left.=[-255,255] ; \forall \frac{\text { output }}{25.5} \bmod =0\right\}$

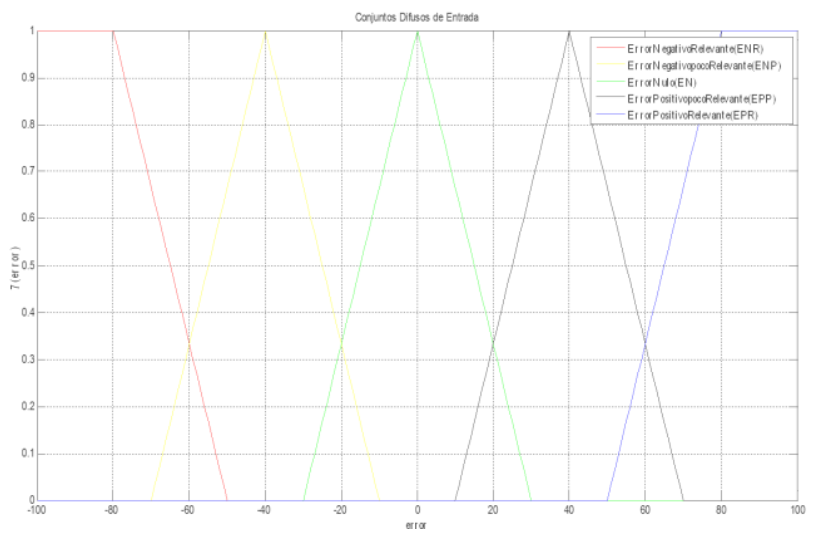

Gráfico 1 Conjuntos de entrada del sistema de inferencia difuso

Fuente: elaboración propia [Matlab] 


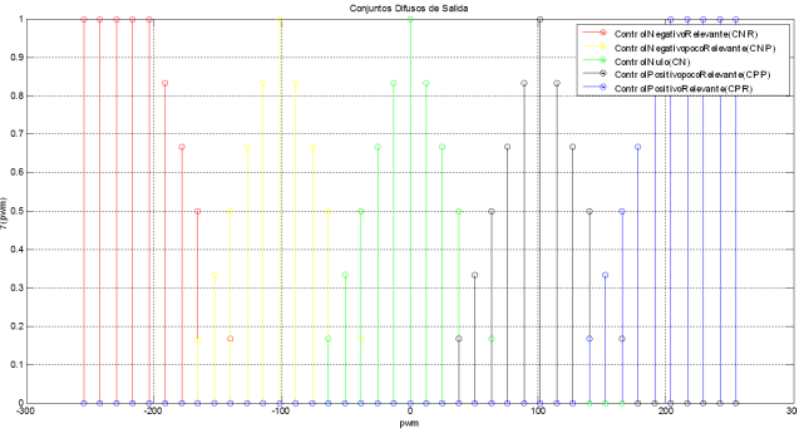

Gráfico 2 Conjuntos de salida del sistema de inferencia difuso

Fuente: elaboración propia [Matlab]

La fuzzificación realiza la abstracción de los datos ingresados al sistema difuso, como parte del conjunto difuso de entrada (Czabanski, Jezewski \& Leski, 2017). Tal grupo de datos ingresan al mecanismo de inferencia, en el que se llevan a cabo la implicación, dadas las reglas de inferencia propuestas y mostradas en la tabla 1, y la agregación. La obtención de un dato de salida es posible a través de la defuzzificación, en la que el valor deducido del proceso anterior y perteneciente al conjunto difuso de salida es traducido para su representación real (Cpalka, 2006). El proceso de fuzzificación se realiza a partir de la implicación de Mamdani, estrategia que relaciona el valor de pertenencia determinado en uno o varios valores específicos de error propuestos para su análisis, una vez aplicado el proceso de fuzzificación, con los valores de pertenencia asociados a los elementos de los conjuntos de salida correspondientes, obteniéndose los valores de pertenencia mínimos entre ambos componentes comparados (Lee, et al., 2010). Mientras la operación de defuzzificación emplea el método de centro de gravedad (COG) el cual permite calcular un valor único o centroide que concentra un promedio de los valores del conjunto final deducido, junto con sus respectivas pertenencias (Cpalka, 2006). Las etapas ejecutadas dentro del sistema de inferencia difusa se visualizan en la figura 2.

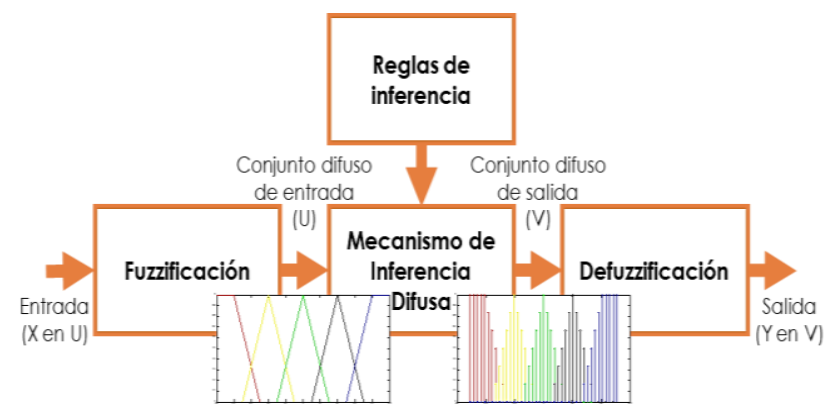

Figura 2 Etapas del sistema de inferencia difusa Fuente: elaboración propia

\begin{tabular}{|l|c|l|}
\hline \multicolumn{2}{|c|}{ Señal de error } & \multicolumn{2}{|c|}{ Señal de control } \\
\hline $\begin{array}{l}\text { Error negativo } \\
\text { relevante }\end{array}$ & $\rightarrow$ & $\begin{array}{l}\text { Control positivo } \\
\text { relevante }\end{array}$ \\
\hline $\begin{array}{l}\text { Error negativo poco } \\
\text { relevante }\end{array}$ & $\rightarrow$ & $\begin{array}{l}\text { Control positivo poco } \\
\text { relevante }\end{array}$ \\
\hline Error negativo nulo & $\rightarrow$ & Control nulo \\
\hline $\begin{array}{l}\text { Error positivo poco } \\
\text { relevante }\end{array}$ & $\rightarrow$ & $\begin{array}{l}\text { Control negativo poco } \\
\text { relevante }\end{array}$ \\
\hline $\begin{array}{l}\text { Error positivo } \\
\text { relevante }\end{array}$ & $\rightarrow$ & $\begin{array}{l}\text { Control negativo } \\
\text { relevante }\end{array}$ \\
\hline
\end{tabular}

Tabla 1 Reglas de inferencia propuestas para el mecanismo de inferencia difusa

Fuente: elaboración propia

Dada una compilación de datos de entrada y salida de un sistema difuso cuyo comportamiento es deseable duplicar, se seleccionó un primer grupo de éstos como parámetros del algoritmo genético. Tal algoritmo entrenará al sistema de inferencia difuso bajo análisis, determinando el valor de los vértices de los conjuntos de entrada y salida requeridos para aproximar la reacción del mismo, según los datos de entrada del conjunto difuso de referencia para imitar su comportamiento. La programación del algoritmo genético ejecuta los procesos presentados en la figura 3 .

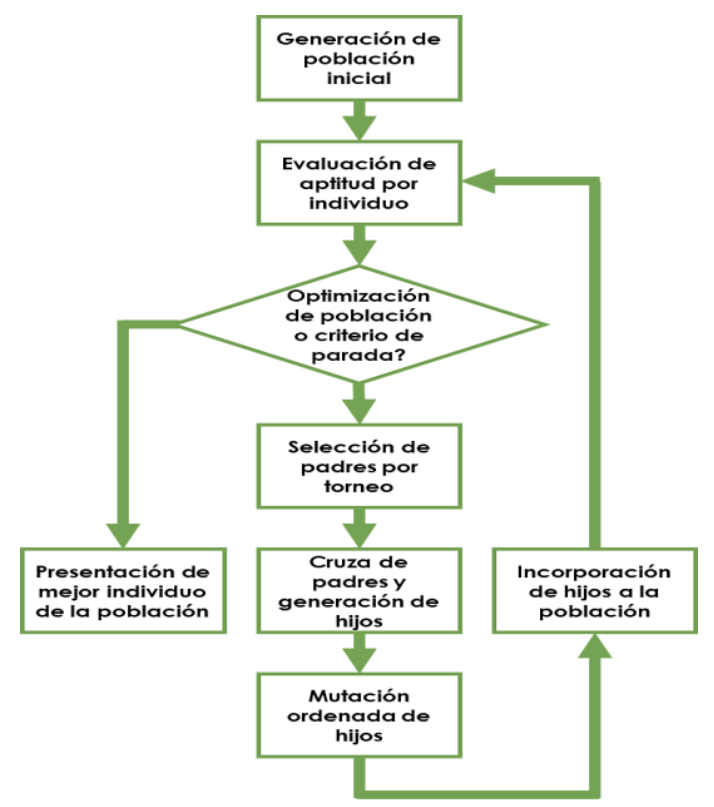

Figura 3 Procesos del algoritmo genético Fuente: elaboración propia

La operación del algoritmo genético principia con la generación de una población inicial binaria, en este caso se propuso el uso de 34 genes, 17 pertenecientes a cada conjunto difuso de entrada o salida establecidos, los cuales representan a los valores de sus vértices, y que a su vez son integrados por 16 alelos.

RODRÍGUEZ-FRANCO, Martín Eduardo, DELGADO-GUERRERO, Sergio Humberto, LÓPEZ-ÁLVAREZ, Yadira Fabiola y JARA-RUIZ, Ricardo. Sintonización genética de sistema de inferencia difuso aplicado al control de posición en un motor de corriente directa. Revista de Innovación Sistemática. 2020 
A partir de tal población, habrá de ser evaluada la aptitud de cada individuo para conocer su adaptabilidad y permanencia en la misma conforme vayan surgiendo nuevas generaciones; la aptitud se determina mediante el cálculo de los mejores valores asignados a los vértices mencionados. Posteriormente, se valora si se ha alcanzado la optimización de la población, es decir, se han obtenido a los mejores individuos de forma global; o se ha cumplido con la condición que permite finalizar la ejecución del algoritmo, que consiste en alcanzar la resolución propuesta en la discrepancia existente entre los conjuntos difusos originales y aquellos generados por el proceso de entrenamiento.

La aplicación de un proceso de selección de individuos por el método de Torneo, permite la determinación de los dos mejores individuos de la población, mismos que son sometidos a un proceso de cruza para generar nuevos individuos con características de ambos padres. Una vez generados tales hijos, su información genética también se ve alterada por efecto de la mutación, una modificación en algunos de los valores correspondientes a sus alelos. Estos nuevos individuos son incorporados en la población, y su aptitud es nuevamente evaluada a fin de descartar a los peores dos elementos, y repetir la totalidad del proceso descrito.

\section{Evaluación de resultados}

Un segundo grupo de datos provenientes de la compilación mencionada fueron empleados para la ejecución de las pruebas de funcionamiento del sistema híbrido constituido. Tales pruebas iniciaron con la aplicación de los valores de los vértices deducidos durante el entrenamiento del sistema de inferencia difuso, mismos que se muestran en el gráfico 3 para los conjuntos de entrada y en el gráfico 4 para los conjuntos de salida.

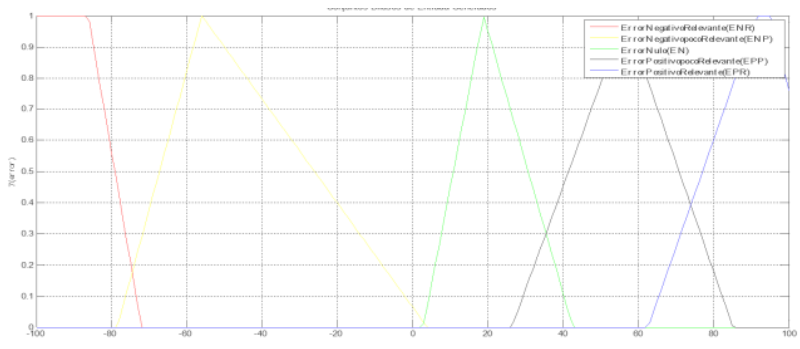

Gráfico 3 Conjuntos de entrada del sistema de inferencia difuso posterior al entrenamiento

Fuente: elaboración propia [Matlab]

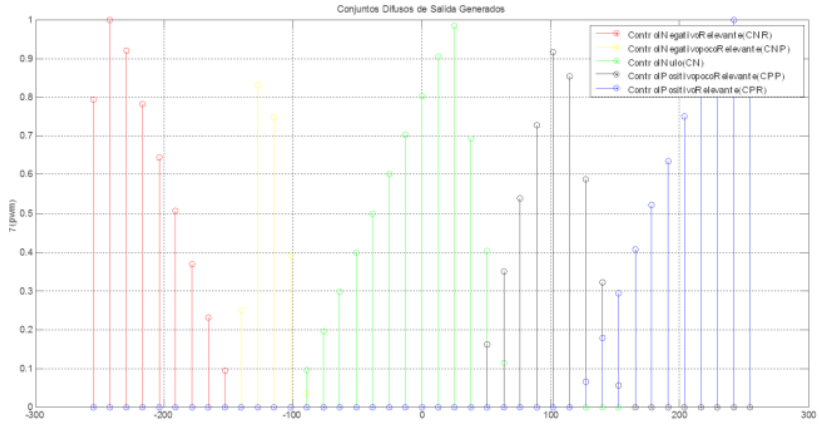

Gráfico 4 Conjuntos de salida del sistema de inferencia difuso posterior al entrenamiento

Fuente: elaboración propia [Matlab]

Además, fueron ingresados al sistema de inferencia difuso entrenado, los datos de prueba previamente establecidos, para obtener el comportamiento del mismo, tanto al aplicar los valores de los vértices en los conjuntos originalmente planteados, así como aquellos obtenidos por acción del proceso de entrenamiento, según se expone en el gráfico 5. Se constata una estrecha similitud entre los comportamientos de los datos de ingresados a sistema entrenado y aquellos arrojados por la aplicación de los valores de los vértices originalmente propuestos.

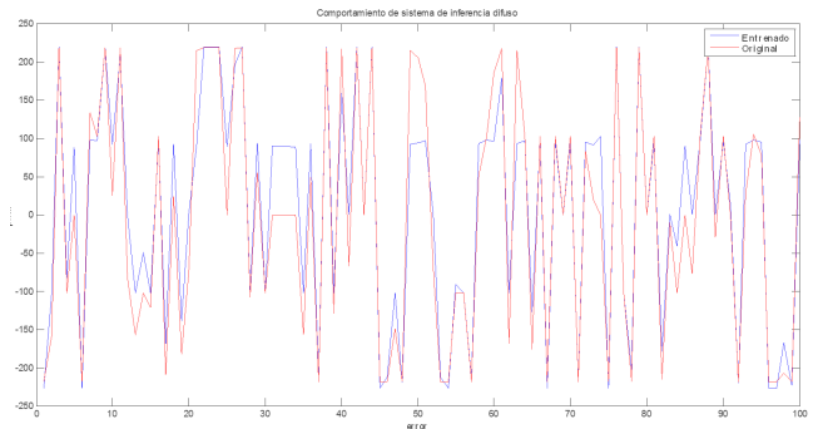

Gráfico 5 Comparación entre comportamientos del sistema de inferencia difuso

Fuente: Elaboración propia [Matlab]

La discrepancia global entre los valores dados de ambos grupos corresponde a 5.49\%, lo cual constata una funcionalidad aceptable del sistema de inferencia difuso sintonizado por algoritmos genéticos.

\section{Conclusiones}

La lógica difusa permite establecer comportamientos específicos de salida, de un sistema bajo estudio, dados ciertos parámetros de entrada; la correlación entre ambos conjuntos se realiza mediante una relación de implicación. 
Los algoritmos genéticos permiten la deducción de valores óptimos que llevan al comportamiento de variables específica de una forma conveniente, al proceso tratado, basado en un proceso evolutivo de éstas.

En el presente estudio, la combinación de ambas técnicas propició la sintonización de un sistema de inferencia difuso dados valores de entrada y salida específicos para el control de posición de un motor de corriente directa. Por lo cual, fue tarea del sistema hibrido deducir los valores estratégicos de los vértices de los conjuntos difusos para lograr resultados muy similares a algunos previamente propuestos.

Al finalizar esta aplicación fue posible caracterizar un error global de $5.49 \%$ entre los datos de salida previos y los arrojados por el sistema sintonizado, lo cual resulta de gran importancia en un sistema de posición al permitir la corrección oportuna de la magnitud del error, dada una consigna, a través de la generación de la señal adecuada hacia el controlador empleado.

\section{Referencias}

Arthur, J., et al. (2019). Genetic algorithm for nuclear data evaluation applied to subcritical neutron multiplication inference benchmark experiments. Annals of Nuclear Energy. 853862.

Bede, B., Rudas, I. J., \& Fodor, J. (2007). Friction model by using fuzzy differential equations. Lecture Notes in Computer Science. 23-32.

Civelek, Z. (2019). Optimization of fuzzy logic (Takagi-Sugeno) blade pitch angle controller in wind turbines by genetic algorithm. Engineering Science and Technology, an International Journal. In Press, Corrected Proof.

Cpalka, K. (2006). A Method for Designing Flexible Neuro-fuzzy Systems. Lecture Notes in Computer Science. 212-219.

Czabanski, R., Jezewski, M., \& Leski, J. (2017). Theory and applications of ordered fuzzy numbers. Studies in Fuzziness and Soft Computing. Cham: Springer International Publishers.
Kesarwani, A., \& Khilar, P. M. (2019). Development of trust based access control models using fuzzy logic in cloud computing. Journal of King Saud University - Computer and Information Sciences. In Press, Corrected Proof.

Kunqi, M., et al. (2019). Numerical investigation on fuzzy logic control energy management strategy of parallel hybrid electric vehicle. Energy Procedia. 2643-2648.

Lee, D. H., et al. (2010). Robust Hळ control for uncertain nonlinear active magnetic bearing systems via Takagi-Sugeno fuzzy models. International Journal of Control, Automation and Systems. 636-646.

Passos, G., \& Barrenechea, M. (2019). Genetic algorithms applied to an evolutionary model of industrial dynamics. EconomiA. In Press Corrected Proof.

Qiao, F., et al. (2004). Adaptive sliding mode control for MIMO nonlinear systems based on fuzzy logic scheme. International Journal of Control, Automation and Systems. 51-62.

Zadeh, L. A. (1999). Computing with words in information/intelligent systems 1. Studies in Fuzziness and Soft Computing. Heidelberg: Springer-Verlag. 